\title{
Second Trial of Low Dosages of DDS in Lepromatous Leprosy
}

\author{
D. L. LEIKER \\ Dermatologist, Royal Tropical Institute, Amsterdam, Netherlands \\ D. CARLING \\ Medical Superintendent, Bornu Provincial Leprosy Settlement \\ Sudan United Mission, Molai, N. Nigeria
}

\begin{abstract}
Three small groups of patients with lepromatous leprosy were treated for 21 months with a low dosage of DDS, receiving respectively 250,50 , and $20 \mathrm{mg}$ of the drug weekly. In all groups the percentage of infiltration and the bacteriological index decreased, as did also the percentage of fragmented bacilli, and in most cases after one year $90 \%$ of the bacilli had become granular. No patient became bacteriologically negative. The frequency of lepromatous reactions remained unchanged in the 2 groups receiving the higher doses, and increased in the group given $20 \mathrm{mg}$ weekly. It is suggested that a weekly dosage of $200 \mathrm{mg}$ of DDS should be adequate for uncomplicated lepromatous leprosy.
\end{abstract}

A previous study by the authors (Leiker and Carling, 1966) suggested that a dosage of 200 or $400 \mathrm{mg}$ of DDS weekly was not significantly less effective than one of 600 to $800 \mathrm{mg}$ weekly. In the present study 3 groups of lepromatous patients ( 14 in all) were treated for 21 months with DDS, 3 of them receiving $250 \mathrm{mg}$ weekly (Group 1), 5 a dose of $50 \mathrm{mg}$ (Group 2) and 6 one of $20 \mathrm{mg}$ of the drug weekly (Group 3).

All the patients were clinically, bacteriologically and histologically lepromatous. Most of them had received previous treatment, but all were still strongly bacteriologically positive when the trial was started. At the beginning of the trial and then every 3 months thereafter smears were taken from 6 sites and examined locally. The bacteriological indices are shown in Table 1.

In addition biopsy specimens were taken every 3 months, each time from the same lesion, near to but just avoiding the scar of previous biopsies. These specimens were assessed blindly, the dosage group to which the patient belonged being unknown to the investigator. Assessment was based on the percentage of sections occupied by infiltration, the bacterial index (B.I.), the percentage of morphologically intact bacilli, the percentage of fragmented bacilli, and the percentage of granular bacilli. Deeply and evenly stained bacilli were recorded as intact and regarded as alive. Bacilli showing a slightly irregular staining but not broken up by unstained parts were recorded as fragmented-a proportion of these bacilli may still have been alive. Bacilli which were broken up into a number of granules were recorded as granular and were regarded as being dead. The results of examination of the biopsy material are shown in Table 2. The frequency of lepromatous reactions occurring during the 2 years immediately before the trial and also those during the trial was recorded; the numbers are shown in Fig. 1 .

\section{RESULTS}

In all 3 groups the percentage of infiltration decreased, the average decrease being $15 \%$ per annum. The differences between the groups in this respect are not significant.

In all groups the B.I. decreased significantly, the decrease being about 1.0 per annum. This is about the same rate of decrease as has been found elsewhere in patients receiving $600 \mathrm{mg}$ of DDS weekly. In the patients treated with 20 to 
TABLE 1

Bacterial index in smears taken at 3-monthly intervals

\begin{tabular}{|c|c|c|c|c|c|c|c|c|c|c|c|c|}
\hline & \multicolumn{8}{|c|}{ Months } & \multirow{2}{*}{$\begin{array}{c}\text { Total dosage } \\
\text { IDDS } \\
(g)\end{array}$} & \multicolumn{3}{|c|}{ No. of reactions } \\
\hline & 0 & 3 & 6 & 9 & 12 & 15 & 18 & 21 & & befor & e trial & trial \\
\hline \multicolumn{13}{|c|}{ Group I-250 mg DI)S weekly } \\
\hline 2007 & 1.65 & 2.15 & 1.65 & 0.83 & $(0.83)$ & 0.83 & 0.5 & 0.6 & 27.35 & & 0 & 0 \\
\hline 2192 & 5.0 & 4.0 & 3.50 & 3.50 & 2.50 & 3.50 & 3.1 & 3.1 & 22.35 & & 0 & 0 \\
\hline 2120 & 5.0 & 5.0 & 3.65 & 4.50 & 3.80 & 4.00 & 4.1 & 4.1 & 23.35 & & 0 & \\
\hline Average & 3.9 & 3.7 & 2.9 & 2.9 & 2.4 & 2.8 & 2.6 & 2.6 & 24.4 & Total & 0 & \\
\hline \multicolumn{13}{|c|}{ Group II-50 mg DDS weekly } \\
\hline 2053 & 2.85 & 3.30 & 3.85 & 2.83 & 3.5 & 3.0 & 1.6 & 1.3 & 8.35 & & 0 & 2 \\
\hline 2057 & 2.85 & 3.85 & 3.35 & 2.66 & 2.5 & 1.1 & 1.5 & 1.1 & 8.80 & & 0 & 0 \\
\hline 2006 & 2.50 & 2.65 & 1.85 & 1.60 & 0.8 & 0.5 & 0.5 & 0.6 & 8.35 & & 2 & 0 \\
\hline 2000 & 1.15 & 2.00 & 1.15 & 1.30 & 1.1 & 0.8 & 0.6 & 0.3 & 8.89 & & 0 & 0 \\
\hline 2158 & 2.50 & 2.50 & 1.85 & 1.50 & 1.1 & 0.6 & 0.5 & 0.5 & 9.15 & & 0 & 0 \\
\hline Average & 2.4 & 2.9 & 2.4 & 2.0 & 1.8 & 1.2 & 0.9 & 0.8 & 8.7 & Total & 2 & 2 \\
\hline \multicolumn{13}{|c|}{ Group III-20 mg DDS weekly } \\
\hline 2067 & 3.65 & 4.0 & 4.0 & 3.5 & 3.1 & 3.6 & 3.0 & 2.6 & 2.6 & & 0 & ] \\
\hline 2043 & 1.00 & 1.15 & 1.5 & 1.16 & 0.8 & 0.3 & () & 0 & 2.6 & & 1 & 0 \\
\hline $2150 \mathrm{P}$ & 3.30 & 3.50 & 3.35 & 3.60 & 3.3 & 3.3 & 2.0 & 2.8 & 2.0 & & 1 & 4 \\
\hline $2137 \mathrm{R}$ & 4.50 & 4.50 & 3.80 & 3.83 & 2.8 & 4.8 & $4.1)$ & $(4.0)$ & 5.0 & & 1 & 5 \\
\hline $2008 \mathrm{R}$ & 2.85 & 2.85 & 2.15 & 2.16 & 1.2 & 1.5 & 1.0 & 0.5 & 4.39 & & 2 & 4 \\
\hline $2003 \mathrm{R}$ & 2.65 & 3.65 & 3.35 & 2.66 & 2.0 & 2.1 & 1.6 & 1.5 & 2.15 & & 7 & 7 \\
\hline Average & 3.0 & 3.3 & 3.0 & 2.8 & 2.2 & 2.6 & 2.0 & 1.9 & 3.12 & $\begin{array}{r}\text { freq } \\
\text { Total }\end{array}$ & $\begin{array}{l}\text { juent } \\
2\end{array}$ & 21 \\
\hline
\end{tabular}

Not included-2246, after 5 months of treatment (B.I. 25 to 5 ) continuous reaction (borderline tuberculoid lesions) steroid dependent, trial stopped. 2248 , abandonment after 9 months.

$\mathrm{R}$, Repeated reactions.

( ), No biopsy taken, figures refer to last biopsy.

$50 \mathrm{mg}$ of DDS weekly the decrease was on the average slightly less in the 2 nd year of treatment than in the lst year.

As most patients had received previous treatment the percentage of intact bacilli was already low at the beginning of the trial. In only one patient a few intact bacilli, accompanied by an increase in the percentage of fragmented bacilli, reappeared after 15 months of treatment. This patient had received $300 \mathrm{mg}$ of I)ISS weekly.

In most patients the percentage of fragmented bacilli began to decrease during the first 3 months of treatment and in all groups a marked decrease was seen in the second 3 months. In most patients in all 3 groups $90 \%$ or more of the bacilli had become granular after one year of treatment. Further increase in the percentage of granular bacilli was slower in all groups. However, none of the patients had become bacteriologically. negative at the end of the trial.

Groups 1 and 2 showed no significant difference in frequency of lepromatous reactions before and during the trial, but in Group 3 the frequency of reactions increased during the trial. 
'T.ABLE : 2

Results of biopsies at 3-monthly intervals

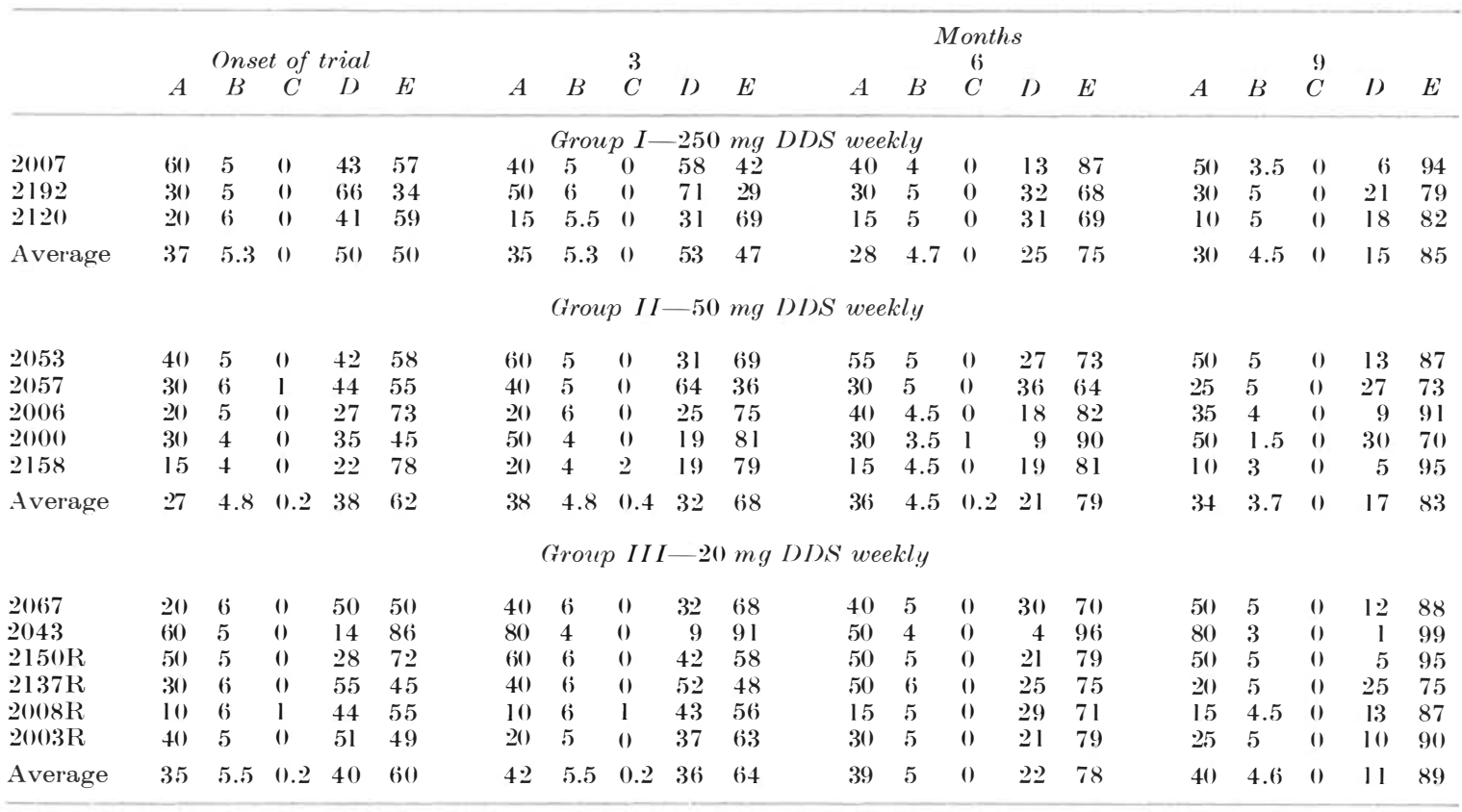

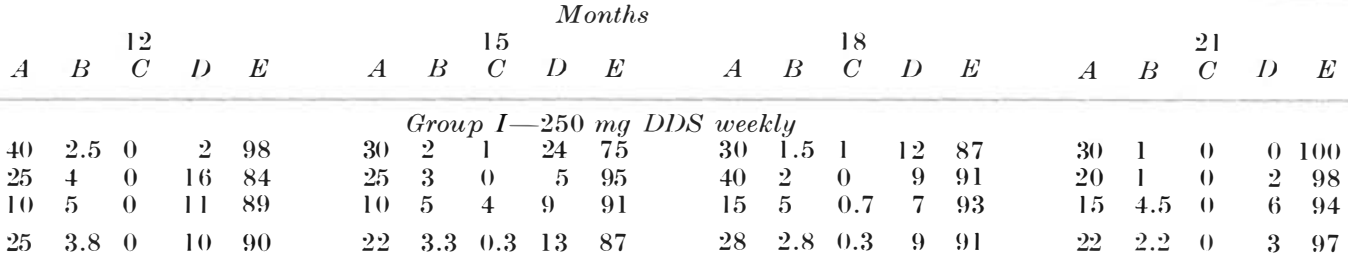

Group II-5() mg I)I)S weekly

\begin{tabular}{|c|c|c|c|c|c|c|c|c|c|c|c|c|c|c|c|c|c|c|c|c|}
\hline 2053 & 60 & 4.5 & () & 6 & 94 & 5() & 4 & () & 3 & 97 & 40 & 5 & 0 & 4 & 96 & 25 & 5 & () & 4 & 96 \\
\hline 2057 & 20 & 5 & () & 5 & 95 & 15 & 5 & 0 & 12 & 88 & 20 & 5.5 & 0 & 17 & 83 & 20 & 5 & 0 & 8 & 92 \\
\hline 2006 & 50 & 4.5 & 0 & 9 & $9 \mathrm{l}$ & 40 & 4 & 0 & 4 & 96 & 45 & 4 & 0 & 10 & 90 & 20 & 2 & 0 & 2 & 98 \\
\hline 2000 & 50 & 2 & 0 & 12 & 88 & 4) & 2 & () & 15 & 85 & 60 & 2 & 0 & 23 & 77 & 20 & 2 & 0 & 2 & 98 \\
\hline 2158 & 10 & 2 & () & l & 99 & 15 & 2 & () & 6 & 94 & 15 & 2 & 0 & 7 & 93 & 5 & 2 & 0 & () & 100 \\
\hline Average & 38 & 3.8 & () & 7 & 93 & 32 & 3.4 & () & 8 & 92 & 36 & 3.7 & () & 12 & 88 & 18 & 3.2 & () & 3 & 97 \\
\hline \multicolumn{21}{|c|}{ Group III-20 $\mathrm{mg}$ DDS weekly } \\
\hline 2067 & 30 & 5 & () & 14 & 86 & 10 & 4.5 & () & 7 & 93 & 5 & 4 & () & 6 & 94 & 10 & 4.5 & () & 4 & 96 \\
\hline 2043 & 70 & 2 & () & 1 & 99 & 5 & 2 & 0 & 1 & 99 & 15 & 2 & () & 2 & 98 & 25 & 1.5 & 0 & 2 & 98 \\
\hline $2150 \mathrm{R}$ & 45 & 5 & () & 7 & 93 & 40 & 5 & () & 9 & 91 & 40 & 5 & () & 7 & 93 & 50 & 4 & () & 2 & 98 \\
\hline $2137 \mathrm{R}$ & 20 & 5 & () & 16 & 84 & 15 & 5 & () & 22 & 78 & $(15)$ & 5 & () & $2 \cdot 2$ & 78) & $(15$ & 5 & () & 22 & 78) \\
\hline $2008 \mathrm{R}$ & 10 & 4 & () & 2 & 98 & 10 & 4 & () & 1 & 99 & 10 & 4 & () & l & 99 & 5 & 4 & () & 1 & 99 \\
\hline $2003 \mathrm{R}$ & 10 & 2 & () & 1 & 99 & I0) & 3.5 & () & 7 & 93 & 10 & 2 & () & 4 & 96 & 10 & 2 & () & () & 100 \\
\hline Average & 31 & 3.9 & () & 7 & 93 & 15 & 4 & 0 & 8 & 92 & 16 & 3.7 & () & 7 & 93 & 20 & 3.5 & () & 5 & 95 \\
\hline
\end{tabular}

A, proportion of of section occupied by infiltrations; B, bacillary index; C, percentage of intact bacilli; $\mathrm{D}$, percentage of fragmented bacilli; $\mathrm{E}$, percentage of granular bacilli; $\mathrm{R}$, repeated reactions. ( ), no biopsy taken, figures refer to last biopsy. 

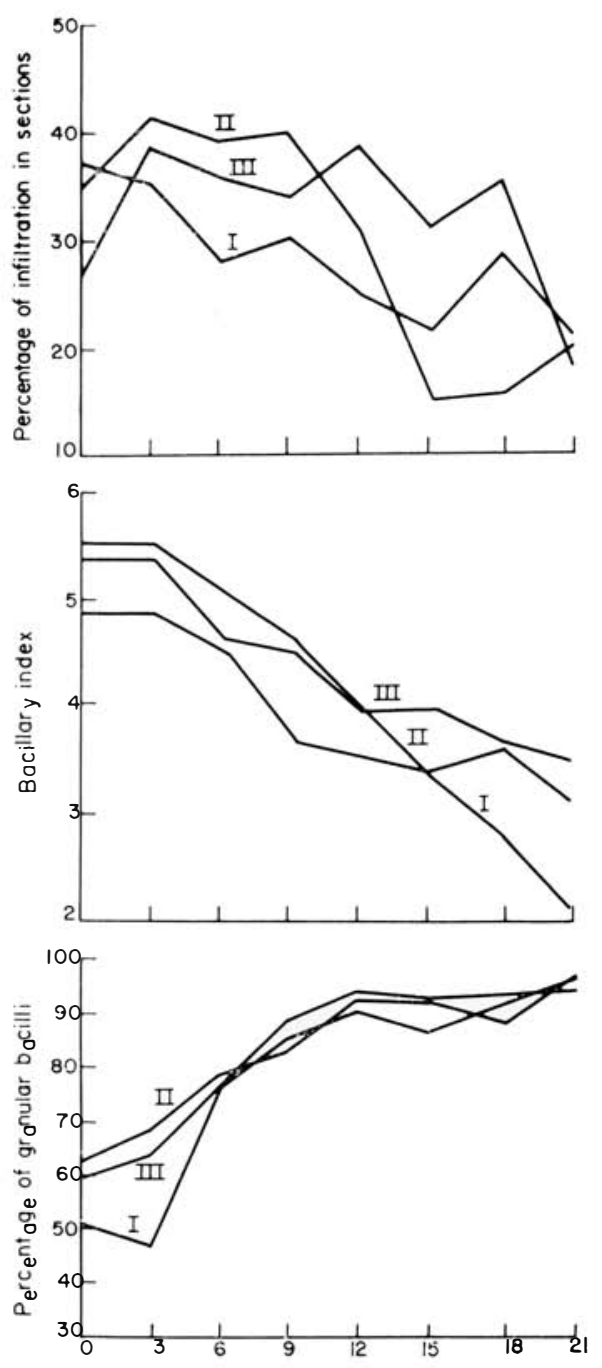

FIG. 1

Low dosage of DDS. Group 1, $250 \mathrm{mg}$, Group 2, $50 \mathrm{mg}$, Group 3, $20 \mathrm{mg}$ of DDS weekly.

\section{DISCUSSION}

The number of patients in the trial was admittedly small. The frequency of detailed observations, however, was high. The bacteriological changes in most patients of all groups showed basically the same trend.

All patients improved during the trial. This improvement, however, cannot be entirely ascribed to the activity of the drug. The shift from fragmented bacilli to granular bacilli and the decrease in the B.I. were probably to a large extent due to removal of dead bacilli from the tissues by the body itself and not due to the effect of the drug. It is, however, significant that during 15 months of treatment intact bacilli did not reappear in any of the patients treated with the lower dosages of DDS, and that after 15 months only a few intact bacilli appeared temporarily in a few patients who had shown frequent reactions. This suggests that even these low dosages do inhibit the multiplication of bacilli. The slightly slower decrease in the percentage of fragmented bacilli found in the first 6 months in the groups treated with 20 and $50 \mathrm{mg}$ of DDS weekly, as compared with the group given $250 \mathrm{mg}$ weekly, suggests that the lower dosages are somewhat less effective than the higher. Until further trials in untreated lepromatous patients or patients with a higher percentage of intact bacilli have produced more information about the exact degree of efficacy of the lower dosages it seems wise to regard a dosage of about $200 \mathrm{mg}$ of DDS weekly as adequate for patients with uncomplicated lepromatous reactions. In patients with frequent reactions the lower dosages may be used, as they are doubtless still efficacious, although the bacteriological effect is possibly not optimal.

In this trial the frequency of reactions was highest in patients receiving the lowest dosage of DDS. This is not surprising because 5 out of the 6 patients in this group had already shown reactions before the trial and the tendency to becoming reactive frequently increases when sulphones, even in a lower dosage, are continued. The outcome of this trial does not show that patients who are intolerant of a high dosage of sulphones will necessarily tolerate a lower dosage. It is possible that patients who have not yet become truly sensitized to sulphones may benefit from a lower dosage, but it is doubtful if the real problem cases will do so in regard to frequency of reactions.

The 4 patients who had frequent reactions while receiving $20 \mathrm{mg}$ of DDS weekly improved bacteriologically, no intact bacilli reappearing in any significant numbers. There is, however, a danger that, although the number of bacilli in the infiltrates, which can be easily demonstrated 
by skin smears and skin biopsies, declines, the bacilli which may be concealed at as yet unidentified sites are not killed by a low dosage of DDS. Such bacilli may become resistant to the drug. The fact that lepromatous patients who have been treated with a higher dosage of DIS not infrequently relapse when treatment is withdrawn soon after skin smears and biopsies have become negative is regarded as evidence that such hidden foci of living bacilli exist and that these bacilli are not easily killed by treatment.

The present trial has not produced a final answer to the question of the most effective dosage of DIS. There is still an urgent need for more detailed studies on the optimal dosage of sulphones.

\section{SUMMARY}

(1) Three groups of lepromatous patients were treated respectively with 250 , 50 or $20 \mathrm{mg}$ of DDS weekly for 21 months.

(2) Bacteriological progress was assessed by examination of 3-monthly smears and by serial biopsies, each time from the same lesion.

(3) All patients improved bacteriologically. The differences in bacteriological progress between the groups were not marked. It is possible that $250 \mathrm{mg}$ of DDS weekly is slightly more effective than the lower dosages.
(4) The frequency of reactions was not significantly lower in patients treated with the lower doses. The highest frequency of reactions was found in patients treated with the lowest dosage but several patients in this group had already shown frequent reactions prior to the trial.

(5) The decrease in bacillary index cannot be ascribed entirely to the effect of the drug, but is partly due to removal of dead bacilli by the body itself. The fact, however, that intact bacilli did not reappear suggests that very low dosages are effective.

(6) Relapse was frequently seen when treatment was stopped soon after smears and skin biopsies had become negative, suggesting that there were hidden foci of living bacilli. As it is not certain that such bacilli too are killed by low dosages and drug resistance may develop, it is suggested that about $200 \mathrm{mg}$ of DDS weekly may be regarded as an adequate dosage for patients with uncomplicated lepromatous leprosy, until further evidence for the efficacy of lower dosages is shown.

\section{REFERENCES}

LEIKER, D. L. and CARLING, D. (1966). Low dosage of DDS. Lepr. Rev. 37, 27. 